\title{
Study of the effect of blurriness in image acquisition for Hand Biometrics in Mobile Devices
}

\author{
Alberto de Santos \\ Group of Biometrics, Biosignals and \\ Security, GB2S \\ Universidad Politécnica de Madrid \\ Campus de Montegancedo \\ Pozuelo de Alarcón, 28223, Madrid \\ Email: alberto@cedint.upm.es
}

\author{
Carmen Sánchez Ávila \\ Group of Biometrics, Biosignals and \\ Security, GB2S \\ Universidad Politécnica de Madrid \\ Campus de Montegancedo \\ Pozuelo de Alarcón, 28223, Madrid \\ Email: csa@cedint.upm.es
}

\author{
Gonzalo Bailador \\ and Javier Guerra \\ Group of Biometrics, Biosignals and \\ Security, GB2S \\ Universidad Politécnica de Madrid \\ Campus de Montegancedo \\ Pozuelo de Alarcón, 28223, Madrid \\ Email: \{gbailador,jguerra\}@ cedint.upm.es
}

\begin{abstract}
This paper presents a study on the effect of blurred images in hand biometrics. Blurred images simulates out-of-focus effects in hand image acquisition, a common consequence of unconstrained, contact-less and platform-free hand biometrics in mobile devices.

The proposed biometric system presents a hand image segmentation based on multiscale aggregation, a segmentation method invariant to different changes like noise or blurriness, together with an innovative feature extraction and a template creation, oriented to obtain an invariant performance against blurring effects.

The results highlight that the proposed system is invariant to some low degrees of blurriness, requiring an image quality control to detect and correct those images with a high degree of blurriness. The evaluation has considered a synthetic database created based on a publicly available database with 120 individuals. In addition, several biometric techniques could benefit from the approach proposed in this paper, since blurriness is a very common effect in biometric techniques involving image acquisition.
\end{abstract}

\section{INTRODUCTION}

Since the new trends in biometrics are oriented to mobile devices, some problems arise directly related to the fact of providing non-invasiveness and contact-less approaches in mobile scenarios.

Concretely, hand biometrics applied to mobile devices involve no platform to place the hand, implying no constraints in hand posing, orientation or openess degree. Therefore, hand biometrics in mobile devices require special efforts in order to avoid inherited problems, mainly blurriness which is a common effect in image acquisition for mobile devices. Blurriness can be defined as the effect which avoids a precise and accurate resolution of the image. This effect can affect directly to the whole accuracy of a biometric system based on hand biometrics.

Therefore, the study of the effect of blurriness in image acquisition and their relation with the performance of the biometric system is the main aim of this study. The proposed algorithm for image segmentation is based on multiscale aggregation, gathering pixels similars in intensity along different scales. In the first scale, similar pixels in intensity are aggregated, forming subgraphs gathering previous information in terms of intensity by means of gaussian functions. This procedure is repeated along several scales until the hand is completely isolated from background. Notice that this proposal requires no knowledge on the background, being very suitable for this scenario in mobile devices. In addition, this algorithm has been properly tested for a standard image segmentation with satisfactory results, and its strength against blurriness will be evaluated within this paper. The evaluation of the paper will be carried out on a synthetic database containing around 500,000 images, considering different degrees of blurriness.

In order to simulate blurriness, different gaussian and laplacian filters are proposed with different parameters so that such an effect can be properly simulated, without requiring a manual database which could not satisfy the requirements of providing different degrees of blurriness. With this procedure, blurriness effect can be measured and controlled, providing a more determined scenario for the experimental layout. The strategy of the paper consists of evaluating the performance of both the segmentation procedure and the biometric system under different degrees of blurriness comparing the results with the performance provided by standard approaches in literature.

The proposed topic can provide a conclusion on the resistance of the hand biometric system to motion or bluriness effects, very common in image acquisition in mobile devices. In other words, the aim of this paper is to evaluate to what extent multiscale aggregation is able to provide an accurate and precise segmentation results and an adequate performance in identifying individuals.

The layout of this paper remains as follows: First of all, a brief literature review is provided in Section II, continuing with the description of the proposed methods in Section IV. The database involved in the evaluation is presented in Section III together with the results in Section V. Finally, conclusions and future work is provided in Section VI.

\section{Literature REVIEW}

Many biometric techniques are based on image acquisition to extract the biometric and personal data. For these biometric 
systems, a precise and accurate image acquisition procedure is essential [1], [2], [3].

In general, a biometric system is formed by different stages, one of them dedicated entirely to evaluate the quality of the acquired image [4], [5]. This image will be assessed, so that a decision on extracting features is carried out. If the image is accepted, features are extracted; otherwise, the image is rejected, and image acquisition is performed again.

There exist several works concerning image quality processing in biometrics most of them related to iris [6], [7], fingerprint [8] or face [9] based biometrics. They evaluate the system in terms of blur, motion, noise or illumination factors, attempting to provide a correction scheme, or reject the image.

Concretely, in hand biometric, some research has been done considering some information on the reliability of the created template [10]. In addition, geometrical features are extracted to ensure that the hand is within the acquired image [11]. However, no specific work has been carried out concerning blur effect in hand image acquisition.

The main contribution of this paper is related to hand image segmentation and invariant feature extraction. Regarding segmentation, there exist many previous work on multiscale aggregation approaches applied to segmentation [12], being specifically applied to hand image segmentation in [13], [14]. In relation to feature extraction, new trends in hand biometrics are focus on applying feature extraction methods invariant to distance to camera, rotation, illumination and other similar changes [15], [16]. These feature extraction techniques are oriented to unconstrained, contact-less hand biometrics, like mobile devices [17] or web-camera devices [15], [18], [19]. Nonetheless, the proposed method will be compared to these former approaches to evaluate to what extent the proposed algorithm is more invariant to changes than the existing methods in literature.

As a conclusion, image quality control has received some attention and research in other biometric techniques, justifying the necessity of exploring a hand biometric technique invariant to blur effects in hand image acquisition.

\section{DATABASE}

This presented methods needs a database with different degrees of blurriness to test both the segmentation algorithm and the feature extraction method. Therefore, we firstly collected a database with real samples, and afterwards, a synthetic database was created considering the same samples as the previous database but with different degrees of blurriness.

The first database contains hand acquisitions of 120 different individuals of an age range from 16 to 60 years old, gathering males and females in similar proportion.

With the aim of a contact-less approach in hand biometrics, hand images were acquired without placing the hand on any flat surface neither requiring any removal of rings, bracelets or watches. Instead, the individual was required to open his/her hand naturally, so the mobile device (an HTC) could take a photo of the hand at $10-15 \mathrm{~cm}$ of distance with the palm facing the camera. This acquisition procedure implies no severe constraints on neither illumination nor distance tomobile camera, being every acquisition carried out under natural light. In addition, it is a database with a huge variability in terms of size, skin color, orientation, hand openness and illumination conditions. In order to ensure a proper feature extraction, independently on segmentation, acquisitions were taken on a defined blue-coloured background, so that segmentation can be easily performed, focusing on hands. Both hands were taken, in a total of two sessions: During the first session, 10 acquisitions from both hands are collected; second session is carried out after 10-15 minutes, collecting again 10 images per hand. The image size provided by the device is $640 \times 340$ pixels. This first database is publicly available at www.gb2s.es.

The synthetic database contains versions of the first database with different degrees of blurriness. The effect of blurriness or out-of-focus is simulated by a gaussian filter applied to each images with different values of dispersion $\sigma$. Concretely, the possible values of $\sigma$ are 2, 5, 10, 20 and 30, covering a wide range of possibilities.

Some examples of this synthetic database are provided in Figure 1.

\section{Methodology}

Thisi section describes the proposed method in detail concerning the segmentation, feature extraction and template creation and matching.

\section{A. Segmentation}

The proposed segmentation algorithm is based on multiscale aggregation [12], [14]. Concretely, the method considers image $I$ as a graph $G=(V, E, W)$, where nodes $v_{i} \in V$ correspond to pixels in image; edges $e_{i, j} \in E$ represent the union between two nodes $v_{i}$ and $v_{j}$; weights $w_{i, j} \in W$ describe the similarity between two nodes $v_{i}$ and $v_{j}$ associated by an edge $e_{i, j}$.

The main contribution of this algorithm is to describe each node as a similarity function based on a specific neighbourhood. In other words, each node $v_{i}$ is described as a function $\phi_{v_{i}}$, assuming a normal distribution $\mathcal{N}(\mu, \sigma)$ in terms of intensities within the 4-neighbour structure. Parameters $\mu$ and $\sigma$ make reference to the average and standard deviation of the intensity in the proposed neighbour structure.

Therefore, the weight $w_{i, j}$ is defined in terms of functions $\phi_{v_{i}}$ and $\phi_{v_{j}}$ as in Equation 1:

$$
w_{i, j}=\int_{\alpha} \sqrt{\phi_{v_{i}} \phi_{v_{j}}} d \alpha
$$

where $\alpha$ represents the color space. In this case, this color space corresponds to CIELAB, concretely the $a$ layer.

The method carries out the following procedure until only two segment remains:

- Obtain set graph $G$ for image $I$

- Order pair of nodes according to weights $W$

- Aggregate nodes in descendent order, based on previous ordering in $W$

- Calculate function $\phi$ for each aggregated segment. 
- Provide neighbour structure applying Delaunay triangulation

Finally, this method comes out with precise and accurate results for hand segmentation [14]. The result section shows that the application of this method together with the feature extraction provides independency against blur effect.

\section{B. Feature Extraction and Template Creation}

After segmentation, tips an valleys within hand are detected [15] in order to established some landmarks points from which geometric features can be extracted.

The feature extraction presented in this method consists of the following steps:

1) Divide the finger from the basis to the tip in $m$ parts and obtain the widths corresponding to each division.

2) Form $n$ groups from previous set of geometrical features, by averaging $\left\lfloor\frac{m}{n}\right\rfloor$ elements in each group.

3) Create set $\Delta_{f_{k}}=\frac{\left\{\delta_{1}^{f_{k}}, \ldots, \delta_{n}^{f_{k}}\right\}}{\bar{\delta}_{f_{k}}}$ where each $\delta_{t}^{f_{k}}$ is defined as the average value of the $t$-th set of elements $\left\lfloor\frac{m}{n}\right\rfloor$, for each finger $f_{k} \in\left\{f_{\text {index }}, f_{\text {middle }}, f_{\text {ring }}, f_{\text {little }}\right\}$. Reader may notice that set $\Delta_{f_{k}}$ is normalized for each finger.

4) Template $\Delta$ contains every set $\Delta_{f_{k}}$ for each finger, with a total of $M=4 \times n$ elements per template.

This steps has described how the distances are extracted and how the template is created for each hand. However, the user template contains more information so that the posterior identification and verification is more precise.

The template is created by considering a total of $N$ samples of an individual. Each sample is a vector of $M$ components extracted form a hand acquisition. The method creates in addition two parameters associated to the template: a vector $\mu_{\text {template }}$ as the average of each element along the $N$ samples; a deviation factor, $\sigma_{\text {template }}$, indicating the deviation of the differences between every pair of samples. In other words, since there are $N$ samples for template creation, a total of $\left(\begin{array}{c}N \\ 2\end{array}\right)$ combinations taking pairs of vectors. From this vector, the euclidean difference is computed and the standard deviation of all vectors is calculated and gathered under $\sigma_{\text {template }}$.

In addition, two more parameters are defined: vector $\pi$ and number $\gamma$. The former vector states which elements within template are less invariant along the $N$ samples. The latter number is defined as in Equation 2:

$$
\gamma=\frac{1}{M}\left(\frac{\mu_{\text {template }}}{\sigma_{\text {template }}} \pi^{T}\right)
$$

This number is related to the coefficient of variation of those samples which are less invariant within the $N$ samples.

Finally, the template $\mathcal{H}$ is created as $\mathcal{H}=$ $\left(\mu_{\text {template }}, \sigma_{\text {template }}, \pi, \sigma\right)$.

\section{Template matching}

One of the main contribution of this paper consists on the template matching. This template comparison is proposed based on a template $\mathcal{H}$ and a certain sample $h$, which can belong to the class associated to $\mathcal{H}$ or to other different class.
Therefore, the probability that sample $h$ belongs to $\mathcal{H}$ is defined by $p(h \mid \mathcal{H})$ in Equation 3:

$$
p(h \mid \mathcal{H})=\frac{1}{M} e^{-a H H^{T}}
$$

where $H$ is defined as in Equation 4

$$
H=\frac{1}{\gamma}\left(\frac{h-\mu_{\text {template }}}{\sigma_{\text {template }}} \circ \pi\right)
$$

where operator $A \circ B=\left[a_{i j} b_{i j}\right]_{\forall i, j}$ is defined as the Hadamard product, an entrywise multiplication for any two matrices $A, B$.

This probability $p(h \mid \mathcal{H})$ is within the interval $[0,1]$, indicating that sample $h$ belongs to user with template $\mathcal{H}$ as $p(h \mid \mathcal{H}) \rightarrow 1$, and vice versa.

Therefore, the biometric verification based on this approach can be carried out by stating a threshold $t h \in[0,1]$, so that an individual (with template $\mathcal{H}_{k}$ ) accesses the system providing a sample $h_{k}$, then the user is correctly verified (authenticated) if $p\left(h_{k} \mid \mathcal{H}_{k}\right) \geq t h$. Otherwise, the user is rejected. Similarly, the identification is considered by considering same previous threshold $t h$, so that, provided a sample of a user, $h_{k}$, the system must decide whom the sample belongs to, or, whether the user is not enrolled in the system. In other words, if $\arg _{i}\left(\min p\left(h_{k} \mid \mathcal{H}_{i}\right) \geq t h\right)$ determines that $i=k$ then the sample $h_{k}$ is properly identified, otherwise the user is not enrolled in the system. Some approaches in literature fail in associating sample $h_{k}$ as a non-existing profile, since they provide the most likelihood an similar class, even if the sample provided by $h_{k}$ corresponds to a non-registered individual [19], [1].

\section{Evaluation AND RESUlts}

The purpose of this section is to evaluate both the segmentation algorithm and the improvement achieved by using the feature extraction method described previously.

The evaluation criteria employed within this paper consider a supervised segmentation assessment, carried out in terms of F-measure [12] and the Equal Error Rate [20], [21], [22].

The evaluation of both algorithms is based on two criteria:

- A supervised segmentation evaluation carried out in terms of F-measure [12]. This factor is able to assess to what extent a segmentation result is accurate in comparison to a ground-truth image.

- The performance of the whole system will be evaluated in terms of Equal Error Rate (EER), comparing the improvement achieved in terms of EER by the proposed method in this paper. The comparison is done to standard approaches like [23].

\section{A. Segmentation Evaluation}

The evaluation of the proposed segmentation algorithm is based on a supervised ground-truth evaluation.

This ground-truth is easy to obtain and reliable in terms of performance due to the distinctive characteristics of the hand 
texture in comparison to the blue background provided on that database.

Thus, the segmentation obtained by the proposed method in the synthetic database will be compared to the result extracted from former database.

Despite of existing several methods to evaluate segmentation in literature, provided a ground-truth, a suitable measure is the F-measure, because of its attributes and properties in terms of sensitivity and specificity [12].

The selected F-measure is defined as the harmonic mean between Precision $(P)$ and Recall $(R)$ as follows in Equation 5:

$$
F=\frac{2 R P}{R+P}
$$

where $P$ (Precision, Confidence) stands for the number of true positives (true segmentation, i.e. classify a hand pixel as hand) in relation to the number of true positives and false negatives (false hand segmentation), and $R$ (Recall, Sensitivity) represents the number of true positives in relation to the number of true positives and false positives (false background segmentation, i.e. consider background as hand). F-measure is within $[0,1]$ interval, so that 0 states a bad segmentation, while on the contrary 1 represents the best segmentation result.

Based on this criteria, segmentation is evaluated in terms of performance, considering F-measure (Equation 5) as the main criterion.

The results are presented in Table I where the segmentation algorithm based on multiscale aggregation has been carried out along different degrees of blurriness. Its performance is compared with simple color-based segmentation [17]. This table I indicates that results obtained by the proposed algorithm methods improves the performance of the color-based segmentation.

The improvement achieved by this method in terms of EER is regarded in next section.

Figure 1 presents a visual evaluation of the segmentation algorithm along different degrees of blurriness. This figure represents samples of the synthetic database with values of $\sigma=\{0,2,5,10,20,30\}$, from left to right. Reader may notice how the performance of the segmentation algorithm decreases as the blurriness effect increases.

\section{B. Performance Evaluation}

The aim of this evaluation consists of assess to what extent the hand biometric system proposed within this paper is independent to changes related to blurriness (outof-focus effect). The samples containing blurriness can be found whether in enrollment (template creation) or in access (template matching), or in both situations. Reader may notice that this paper assumes that the biometric system has no image quality control, deciding if feature extraction can be extracted from a specific image.

Since the samples can be found in both stages of the biometric system, this evaluation regards this possibility by simulating three different scenarios:

\begin{tabular}{ccccccc}
\hline \hline & $0 \%$ & $10 \%$ & $20 \%$ & $50 \%$ & $75 \%$ & $100 \%$ \\
\hline Standard Approach & 4.1 & 5.9 & 7.3 & 10.1 & 13.5 & 15.2 \\
Proposed Approach & 2.5 & 3.1 & 4.7 & 5.2 & 6.4 & 6.7 \\
\hline \hline
\end{tabular}

TABLE II

EQUAL ERROR RATE VARIATION (\%) IN RELATION TO THE PROPORTION OF BLURRED IMAGES IN TRAINING STAGE ONLY. THE PROPOSED METHOD IS MORE INVARIANT TO THE PROPORTION OF BLURRED IMAGES IN A DATABASE THAN STANDARD APPROACHES [23]. THIS RESULTS WERE OBTAINED WITH $\sigma=10$.

\begin{tabular}{ccccccc}
\hline \hline & $0 \%$ & $10 \%$ & $20 \%$ & $50 \%$ & $75 \%$ & $100 \%$ \\
\hline Standard Approach & 4.1 & 5.2 & 6.9 & 9.8 & 14.7 & 18.1 \\
Proposed Approach & 2.5 & 2.9 & 3.8 & 4.2 & 5.1 & 6.3 \\
\hline \hline
\end{tabular}

TABLE III

EQUAL ERROR RATE VARIATION (\%) IN RELATION TO THE PROPORTION OF BLURRED IMAGES IN TESTING STAGE ONLY. THE PROPOSED METHOD IS MORE INVARIANT TO THE PROPORTION OF BLURRED IMAGES IN A DATABASE THAN STANDARD APPROACHES [23]. THIS RESULTS WERE OBTAINED WITH $\sigma=10$.

- First scenario will consider the inclusion of blurred samples only during the training/enrollment stage.

- Second scenario will consider to include blurred samples only during the access (template matching) stage.

- Third scenario regards blurred samples in both stages.

The inclusion of blurred samples within the biometric system is evaluated gradually. The performance of the biometric system will be evaluated along different percentages of blurred images in the database. In other words, the performance will be tested when, for instance, $10 \%$ of the samples are blurred images. Afterwards, this percentage is incremented till $100 \%$ of the database is completely blurred.

The results of the first scenario can be seen in Table II where the Equal Error Rate of the biometric system is obtained along different proportions of blurred image samples in the database. The percentage corresponds to the amount of blurred samples during the training stage. This results were obtained with $\sigma=10$.

The results of the second scenario are presented in Table III where the Equal Error Rate of the biometric system is obtained along different proportions of blurred image samples in the database. Similarly, the percentage corresponds to the amount of blurred samples during the testing stage. This results were also obtained with $\sigma=10$.

From both Tables II and III, it can be concluded that the proposed approach in this paper outperforms standard approaches in terms of identification accuracy (EER), although the proposed method is more sensitive to blurred images in training stage than in testing.

In addition, Table IV contains the relation between EER and $\sigma$. Although the proposed approach is extensively more invariant to blurriness than standard approaches, the effect of great degrees of blurriness also affect the proposed method. Therefore, as future work, we would like to include an image quality control, so that images with great degree of blurriness 


\begin{tabular}{lccccc}
\hline \hline & $\sigma=2$ & $\sigma=5$ & $\sigma=10$ & $\sigma=20$ & $\sigma=30$ \\
\hline Color-based Segmentation & 99.2 & 95.2 & 88.1 & 80.4 & 77.3 \\
Multiscale Aggregation & 99.6 & 97.4 & 91.3 & 85.7 & 82.5 \\
\hline \hline
\end{tabular}

TABLE I

F-MEASURE (\%)FOR THE COMPARED METHODS: COLOR-BASED SEGMENTATION AND MULTISCALE AGGREGATION APPROACH. THE F-MEASURE IS AFFECTED BY BLURRINESS, BUT IT IS MORE NOTORIOUS ON COLOR-BASED SEGMENTATION. IN OTHER WORDS, SEGMENTATION BASED ON MULTISCALE AGGREGATION IS LESS INVARIANT TO BLURRINESS THAN COLOR-BASED SEGMENTATION.

\begin{tabular}{cccccc}
\hline \hline & $\sigma=2$ & $\sigma=5$ & $\sigma=10$ & $\sigma=20$ & $\sigma=30$ \\
\hline Standard Approach & 4.8 & 8.1 & 17 & 20.1 & 23.4 \\
Proposed Approach & 3.2 & 4.2 & 7.1 & 12.3 & 15.2 \\
\hline \hline
\end{tabular}

TABLE IV

EQUAL ERROR RATE VARIATION (\%) IN RELATION TO THE BLURRINESS MAIN PARAMETER, $\sigma$. THE PROPOSED METHOD IS MORE INVARIANT TO BLURRINESS THAN STANDARD APPROACHES [23].

are corrected or rejected.

Finally, the results from third scenario are presented in Table V, where dependency of EER different proportions of blurred images in both training and testing stages. In a normal scenario, it can be assumed that less than $10 \%$ of the images will be blurred, presented within this interval a competitive result in terms of identification accuracy.

\section{CONClusions AND Future WORK}

The main aim of this paper consists of studying the effect of blurred images on the performance of a hand biometric system. Blurriness simulates the out-of-focus effect in hand acquisition, which is a common consequence of unconstrained and contact-less approaches in hand biometrics in mobile devices.

The proposed biometric system attempts to offer independency to this effect, so that the biometric performance remains invariant to some extent, even if some hand samples present a certain degree of blurriness. The aim of this biometric system is to provide more acceptability, by reducing the requirements in image acquisition in terms of image quality.

Main contribution of this paper is two fold: First, a segmentation algorithm based on multiscale aggregation is presented as a proper method to avoid blurriness during segmentation. Secondly, a feature extraction algorithm based on the creation of a template, containing only information/data from one single individual. This feature extraction is based on geometric distances, being invariant to changes like distance to camera, rotation, pose and some degree of blurriness.

The results show that the proposed algorithm improves the performance when compared to standard approaches in literature. However, the performance of the biometric system would remain more invariant with a quality control procedure. In other words, the proposed biometric system is invariant to low degrees of blurriness, requiring a image rejection algorithm for those images with a high degree of blurriness.

As future work, we consider the comparison of the segmentation algorithm to other existing segmentation techniques in hand biometrics, and the research on a image quality control able to detect and correct blurriness effect. In addition, a deeper understanding of the effect of blurriness in other biometric techniques, for instance, iris recognition, remains as future work.

Finally, we will consider other effects in hand image acquisition like motion, similar to blurriness but with oriented gaussian distorsion.

\section{ACKNOWLEDGMENT}

This research has been supported by the Ministry of Industry, Tourism and Trade of Spain, in the framework of the project CENIT-Segur@, reference CENIT-2007 2004.

\section{REFERENCES}

[1] M. Adán, A. Adán, A. S. Vázquez, and R. Torres, "Biometric verification/identification based on hands natural layout," Image Vision Comput., vol. 26, pp. 451-465, April 2008. [Online]. Available: http://portal.acm.org/citation.cfm?id=1332135.1332289

[2] E. Kukula, S. Elliott, B. Gresock, and N. Dunning, "Defining habituation using hand geometry," in Automatic Identification Advanced Technologies, 2007 IEEE Workshop on, 7-8 2007, pp. 242-246.

[3] A. Kumar and D. Zhang, "Personal recognition using hand shape and texture," Image Processing, IEEE Transactions on, vol. 15, no. 8, pp. $2454-2461$, aug. 2006.

[4] E. Kukula and S. Elliott, "Implementation of hand geometry at purdue university's recreational center: an analysis of user perspectives and system performance," in Security Technology, 2005. CCST '05. 39th Annual 2005 International Carnahan Conference on, 11-14 2005, pp. $83-88$.

[5] B. Fernandez-Saavedra, J. Liu-Jimenez, and C. Sanchez-Avila, "Quality measurements for iris images in biometrics," in EUROCON, 2007. The International Conference on 34; Computer as a Tool 34; , sept. 2007, pp. $759-764$

[6] P. Radu, K. Sirlantzis, G. Howells, S. Hoque, and F. Deravi, "On combining information from both eyes to cope with motion blur in iris recognition," in Soft Computing Applications (SOFA), 2010 4th International Workshop on, july 2010, pp. $175-181$.

[7] E. Krichen, S. Garcia-Salicetti, and B. Dorizzi, "A new probabilistic iris quality measure for comprehensive noise detection," in Biometrics: Theory, Applications, and Systems, 2007. BTAS 2007. First IEEE International Conference on, sept. 2007, pp. 1-6.

[8] H. Fronthaler, K. Kollreider, and J. Bigun, "Automatic image quality assessment with application in biometrics," in Computer Vision and Pattern Recognition Workshop, 2006. CVPRW '06. Conference on, june 2006, p. 30.

[9] R.-L. Hsu, J. Shah, and B. Martin, "Quality assessment of facial images," in Biometric Consortium Conference, 2006 Biometrics Symposium: Special Session on Research at the, 19 2006-aug. 21 2006, pp. 1 -6.

[10] A. Kumar and D. Zhang, "Improving biometric authentication performance from the user quality," Instrumentation and Measurement, IEEE Transactions on, vol. 59, no. 3, pp. $730-735$, march 2010.

[11] J. Burgues, J. Fierrez, D. Ramos, M. Puertas, and J. Ortega-Garcia, "Detecting invalid samples in hand geometry verification through geometric measurements," in Emerging Techniques and Challenges for Hand-Based Biometrics (ETCHB), 2010 International Workshop on, aug. 2010, pp. $1-6$. 


\begin{tabular}{|c|c|c|c|c|c|c|c|}
\hline \multicolumn{2}{|c|}{} & \multicolumn{7}{|c|}{ Testing } \\
\cline { 3 - 8 } \multicolumn{2}{|c|}{} & $0 \%$ & $10 \%$ & $20 \%$ & $50 \%$ & $75 \%$ & $100 \%$ \\
\hline \multirow{4}{*}{ Training } & $0 \%$ & 2.5 & 2.9 & 3.8 & 4.2 & 5.1 & 6.3 \\
\cline { 2 - 8 } & $10 \%$ & 3.1 & 3.3 & 4.1 & 4.7 & 5.3 & 6.4 \\
\cline { 2 - 8 } & $20 \%$ & 4.7 & 4.8 & 5.2 & 5.3 & 5.6 & 6.4 \\
\cline { 2 - 8 } & $50 \%$ & 5.2 & 5.4 & 5.7 & 5.7 & 5.8 & 6.5 \\
\cline { 2 - 8 } & $75 \%$ & 6.4 & 6.3 & 6.4 & 6.5 & 6.7 & 6.8 \\
\cline { 2 - 8 } & $100 \%$ & 6.7 & 6.7 & 6.8 & 6.9 & 6.9 & 7.1 \\
\hline
\end{tabular}

TABLE V

DISTRIBUTION OF EER (\%) WITH DIFFERENT PROPORTIONS OF BLURRED IMAGES ALONG TRAINING AND TESTING. THESE RESULTS WERE OBTAINED WITH A BLURRED EFFECT OF $\sigma=10$. THE PROPOSED METHOD IS STILL COMPETITIVE EVEN WITH PLENTY OF BLURRED IMAGES IN BOTH TRAINING AND TESTING.

[12] S. Alpert, M. Galun, A. Brandt, and R. Basri, "Image segmentation by probabilistic bottom-up aggregation and cue integration," Pattern Analysis and Machine Intelligence, IEEE Transactions on, vol. PP, no. 99, p. 1, 2011.

[13] A. Garcia-Casarrubios Munoz, C. Sanchez Avila, A. de Santos Sierra and J. Guerra Casanova, "A mobile-oriented hand segmentation algorithm based on fuzzy multiscale aggregation," in Advances in Visual Computing, ser. Lecture Notes in Computer Science, G. Bebis, R. Boyle, B. Parvin, D. Koracin, R. Chung, R. Hammoud, M. Hussain, T. KarHan, R. Crawfis, D. Thalmann, D. Kao, and L. Avila, Eds. Springer Berlin / Heidelberg, 2010, vol. 6453, pp. 479-488.

[14] A. G.-C. Munoz, A. de Santos Sierra, C. S. Avila, J. G. Casanova, G. B. del Pozo, and V. J. Vera, "Hand biometric segmentation by means of fuzzy multiscale aggregation for mobile devices," aug. 2010, pp. $1-6$.

[15] A. Morales, M. Ferrer, J. Alonso, and C. Travieso, "Comparing infrared and visible illumination for contactless hand based biometric scheme," in Security Technology, 2008. ICCST 2008. 42nd Annual IEEE International Carnahan Conference on, oct. 2008, pp. $191-197$.

[16] A. Kumar and D. Zhang, "Hand-geometry recognition using entropybased discretization," Information Forensics and Security, IEEE Transactions on, vol. 2, no. 2, pp. $181-187$, june 2007.

[17] A. de Santos Sierra, J. Casanova, C. Avila, and V. Vera, "Silhouettebased hand recognition on mobile devices," in Security Technology, 2009. 43rd Annual 2009 International Carnahan Conference on, oct. 2009, pp. $160-166$.

[18] V. Kanhangad, A. Kumar, and D. Zhang, "Contactless and pose invariant biometric identification using hand surface," Image Processing, IEEE Transactions on, vol. 20, no. 5, pp. 1415 -1424, may 2011.

[19] J. Doublet, O. Lepetit, and M. Revenu, "Contactless hand recognition based on distribution estimation," in Biometrics Symposium, 2007, sept. 2007, pp. $1-6$.

[20] R. Sanchez-Reillo, C. Sanchez-Avila, and A. Gonzalez-Marcos, "Biometric identification through hand geometry measurements," Pattern Analysis and Machine Intelligence, IEEE Transactions on, vol. 22, no. 10 , pp. 1168 - 1171 , oct 2000 .

[21] A. Jain and N. Duta, "Deformable matching of hand shapes for user verification," in Image Processing, 1999. ICIP 99. Proceedings. 1999 International Conference on, vol. 2, oct. 1999, pp. 857 -861 vol.2.

[22] A. Kumar, D. Wong, H. Shen, and A. Jain, "Personal verification using palmprint and hand geometry biometrics," in Proceedings of the fourth International Conference on audio- and video-based biometric personal authentication, 2003.

[23] N. Duta, "A survey of biometric technology based on hand shape," Pattern Recogn., vol. 42, pp. 2797-2806, November 2009. [Online]. Available: http://portal.acm.org/citation.cfm?id=1563046.1563065

\section{VITA}

Alberto de Santos Sierra ad gradum promotus fuit in Escuela Técnica Superior de Telecomunicaciones (ETSIT) Matritense. Ad exitum studios duxit in Vrije Universiteit Amsterdam, in Hollandia, Iulio mense, anno Domini MMVII. A mense Septembre laborat Centro Domótica Integral, CeDInt, Universitatis Polithecnicæ Matritensis. Biometria Stresæ, Systematis Reagnitionis Biometricæ et Cryptographia eius inves- tigationis materia est. Nunc doctoris laurea inaugurandus est in ETSIT.

He received the degree of Telecommunication Engineer in Escuela Técnica Superior de Telecomunicaciones (ETSIT), finishing his studies in Vrije Universiteit Amsterdam with a Final Master Project based on Iris Recognition, in July, 2007. At present, he researches in Centro de Domótica Integral, CeDInt, in topics related to Biometric Recognition Systems, Stress Biometry and Cryptography. $\mathrm{He}$ is currently a $\mathrm{PhD}$ Student in ETSIT.

Carmen Sánchez Ávila received the PhD degree in Mathematical Sciences from the Polytechnic University of Madrid, Spain, in 1993. Since 1985 she has been with the Department of Applied Mathematics, Polytechnic University of Madrid, where she conducts research on Digital Signal Processing, Cryptography and Biometric. At present she is Professor in the above mentioned Department where she has been teaching different undergraduate courses related to Mathematical and Numerical Analysis as well as graduate courses in Wavelets in Signal Processing and Biometrics Systems. Her interests in R\&D are still Cryptography, Biometrics and Security Systems.

Gonzalo Bailador del Pozo received his Ph.D. degree in Computer Science from Polytechnical University of Madrid. $\mathrm{He}$ is currently working in the research group GB2S focused on applying pattern recognition techniques to the analysis of temporal signals and mass spectrometry data. His research interests include gesture recognition, gait recognition, odour identification and robotics.

Javier Guerra Casanova He was born in Madrid, studying Telecommunication Engineering in Polytechnical University of Madrid, receiving their master degree in 2008. At present, he is a PhD student whose Doctoral Thesis is related to biometric identification by means of in-air signatures carried out with devices embedding an accelerometer. 

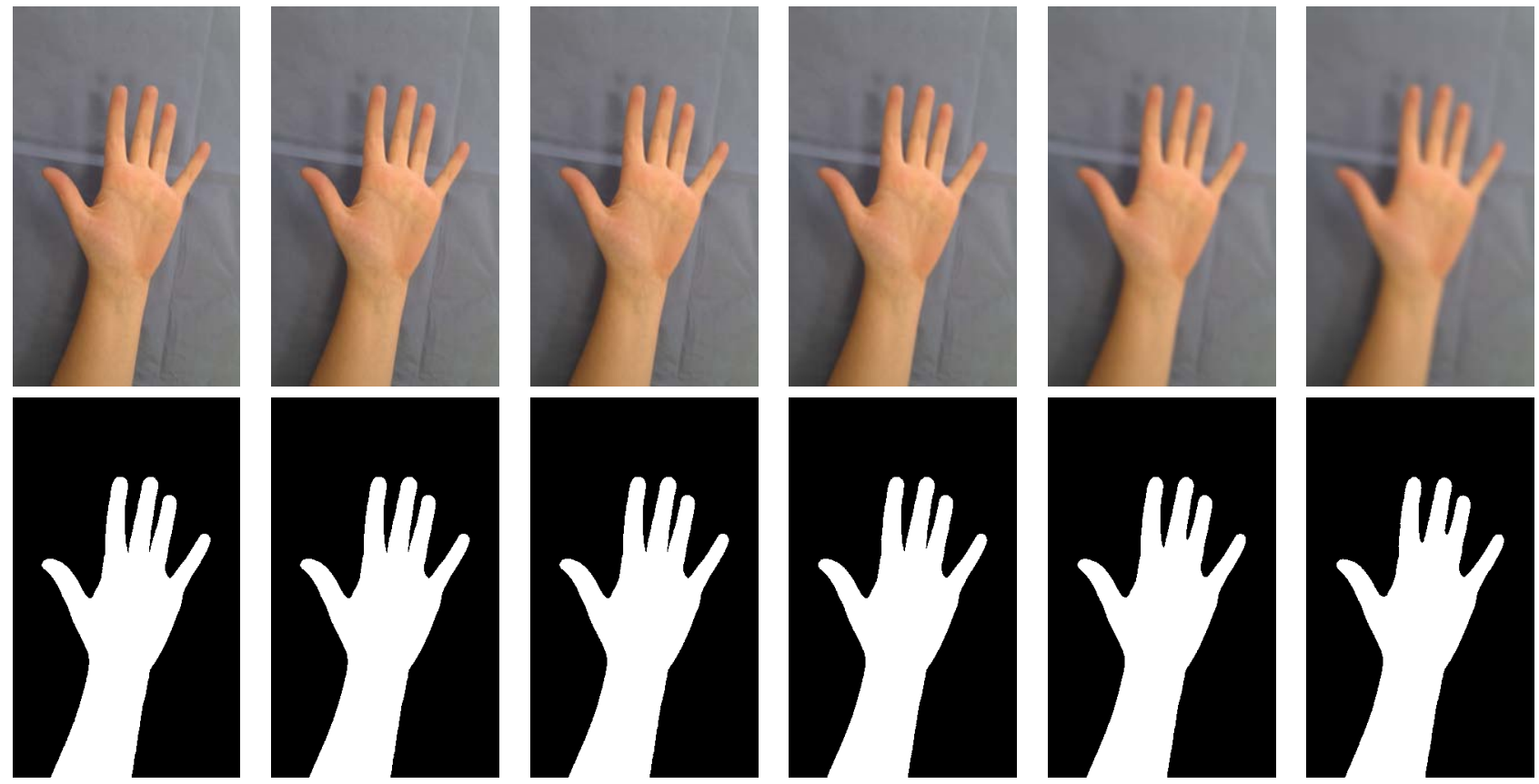

Fig. 1. Samples of the synthetic databases for values of $\sigma=\{0,2,5,10,20,30\}$, respectively. In addition, the segmentation result provided by the multiscale aggregation approach is provided. Notice how the segmentation accuracy decreases as $\sigma$ increases. 